\title{
The Role of the Nucleus Accumbens in Instrumental Conditioning: Evidence of a Functional Dissociation between Accumbens Core and Shell
}

\author{
Laura H. Corbit, ${ }^{1}$ Janice L. Muir, ${ }^{2}$ and Bernard W. Balleine ${ }^{1}$ \\ ${ }^{1}$ Department of Psychology, University of California Los Angeles, Los Angeles, California 90095, and 2School of \\ Psychology, Cardiff University, Cardiff, CF10 3YG, United Kingdom
}

In three experiments we examined the effect of bilateral excitotoxic lesions of the nucleus accumbens core or shell subregions on instrumental performance, outcome devaluation, degradation of the instrumental contingency, Pavlovian conditioning, and Pavlovian-instrumental transfer. Rats were food deprived and trained to press two levers, one delivering food pellets and the other a sucrose solution. All animals acquired the lever-press response although the rate of acquisition and overall response rates in core-lesioned animals were depressed relative to that in the shell- or sham-lesioned animals. Furthermore, in shell- and sham-lesioned rats, post-training devaluation of one of the two outcomes using a specific satiety procedure produced a selective reduction in performance on the lever that, in training, delivered the prefed outcome. In contrast, the core-lesioned rats failed to show a selective devaluation effect and reduced responding on both levers. Subsequent tests revealed that these effects of core lesions were not caused by an impairment in their ability to recall the devalued outcome, to discriminate the two outcomes, or to encode the instrumental action-outcome contingencies to which they were exposed. Additionally, the core lesions did not have any marked effect on Pavlovian conditioning or on Pavlovian-instrumental transfer. Importantly, although shell-lesioned rats showed no deficit in any test of instrumental conditioning or in Pavlovian conditioning, they failed to show any positive transfer in the Pavlovian-instrumental transfer test. This double dissociation suggests that nucleus accumbens core and shell differentially mediate the impact of instrumental and Pavlovian incentive processes, respectively, on instrumental performance.

Key words: nucleus accumbens; core; shell; instrumental conditioning; Pavlovian conditioning; Pavlovian-instrumental transfer; devaluation; contingency; incentive; reward
The neural processes mediating reward and reinforcement have received considerable research attention (Robbins and Everitt, 1996). However, these studies have generally been conducted without consideration of how these reward processes interact with structures that mediate instrumental behavior, and as a consequence, their role in instrumental learning remains to be identified.

Several lines of evidence suggest that the nucleus accumbens (NAC) may play an important role in relating reward processes to the action-outcome associations that underlie instrumental learning (cf. Colwill and Rescorla, 1986; Dickinson and Balleine, 1994). Anatomical studies have revealed that the NAC receives excitatory glutamatergic afferents from "limbic" structures such as the basolateral amygdala, the ventral subiculum, and the medial prefrontal cortices (Powell and Leman, 1976; Kelley and Domesick, 1982; Kelley et al., 1982; Alheid and Heimer, 1988). It also receives dopaminergic inputs from the ventral tegmental area and is a major component of the mesolimbic dopamine system (Moore and Bloom, 1978). On the basis of such anatomical findings, Mogenson and colleagues advanced the influential suggestion that the NAC may be a central structure in the control of action, reflecting an area of limbic-motor integration (Mogen-

Received Nov. 17, 2000; revised Jan. 25, 2001; accepted Feb. 8, 2001.

This research was supported by the National Institute of Mental Health Grant 56446 to B.W.B. We thank Sandra Cetl for her assistance with data collection.

Correspondence should be addressed to Laura Corbit, University of California Los Angeles, Department of Psychology, Box 951563, Los Angeles, CA 90095. E-mail: corbit@ucla.edu.

Copyright (C) 2001 Society for Neuroscience $0270-6474 / 01 / 213251-10 \$ 15.00 / 0$ son et al., 1980, 1988; Mogenson and Yim, 1991). From this perspective, limbic inputs to the accumbens carrying associative and motivational information were argued to influence motor activity via an accumbens-pallidal pathway and the projections of the latter structure to the mesencephalic locomotor region (Groenewegen and Russchen, 1984; Nauta and Domesick, 1984; Haber et al., 1985).

The NAC itself is a heterogeneous structure and can be further divided into anatomically distinct core and shell subregions (Zahm and Brog, 1992). Both core and shell receive inputs from the amygdala, globus pallidus, and ventral pallidum. However, they differ in the density of their cortical afferents; the core receives projections predominantly from the prelimbic, anterior cingulate, and dorsal agranular insular cortices, and the shell receives projections predominantly from infralimbic, ventral agranular insular, and piriform cortices (Zahm and Brog, 1992; Zahm, 2000). Perhaps more important, the efferents of these regions differ substantially; the core projects to conventional basal ganglia circuitry including the ventral pallidum, globus pallidus, and the substantia nigra, whereas the shell projects to subcortical limbic structures such as the lateral hypothalamus, the ventral tegmental area, and the ventromedial ventral pallidum (Zahm and Brog, 1992). These differences in connections raise the possibility that these two regions of the accumbens may be functionally independent.

A number of studies have demonstrated that lesions of the NAC act to reduce instrumental performance (Balleine and Killcross, 1994), a finding that has been confirmed using more selective manipulations of glutamatergic (Kelley et al., 1997) and 
dopaminergic (Sokolowski and Salamone, 1998) activity. The general aim of the current study was to characterize more fully the role of the NAC in instrumental conditioning. Specifically, the impact of lesions of the NAC core and shell on instrumental behavior after outcome devaluation, after contingency degradation, and in a Pavlovian-instrumental transfer test was examined.

\section{MATERIALS AND METHODS}

\section{Experiment 1: outcome devaluation by specific satiety} Subjects and apparatus

The subjects were 24 experimentally naïve female Long-Evans rats. The rats were housed singly and were handled daily for 1 week before surgery. Training and testing took place in 16 Med Associates (East Fairfield, VT) operant chambers housed within sound- and light-resistant shells. Each chamber was equipped with a pump fitted with a syringe that delivered $0.1 \mathrm{ml}$ of a $20 \%$ sucrose solution into a recessed magazine in the chamber. Each chamber was also equipped with a pellet dispenser that delivered one $45 \mathrm{mg}$ Noyes pellet (formula A/I) when activated. The chambers contained two retractable levers that could be inserted to the left and right of the magazine. A $3 \mathrm{~W}, 24 \mathrm{~V}$ house light mounted on the top-center of the wall opposite the magazine provided illumination. Microcomputers equipped with the MED-PC program (Med Associates) controlled the equipment and recorded the lever presses.

\section{Surgery}

At the time of surgery, animals weighed between 266 and 338 gm. There were three surgical groups; rats received cell body lesions of either the core or shell region of the nucleus accumbens or sham surgery. Rats were anesthetized using sodium pentobarbital (Nembutal, $50 \mathrm{mg} / \mathrm{kg}$ ), treated with atropine $(0.1 \mathrm{mg})$, and then placed in a stereotaxic frame (Stoelting Company, Wood Dale, IL) with the incisor bar set at $-3.3 \mathrm{~mm}$. The scalp was retracted to expose the skull, and small burr holes were drilled above the target regions. For lesions of the core $(n=7)$, animals received bilateral injections of $0.5 \mu \mathrm{l}$ of $0.12 \mathrm{M}$ NMDA in two sites (one per side) using a $1 \mu$ l Hamilton syringe (all coordinates in millimeters relative to bregma; anteroposterior, +1.2; mediolateral, \pm 2.1 ; and dorsoventral, $-7.0)$. Each injection was made over $2 \mathrm{~min}$ and allowed to diff use for an additional $2 \mathrm{~min}$ before removal of the needle. For lesions of the shell region $(n=9)$, animals received $0.2 \mu$ injections of $0.015 \mathrm{M} \mathrm{AMPA}$ hydrobromide at four sites (two per side, anteroposterior, +1.6; mediolateral, \pm 0.8 ; and dorsoventral, $-6.8,-6.0$ ). Injections were again made over 2 min with an additional 2 min allowed before any movement of the needle. Animals in the surgical control group $(n=8)$ underwent similar treatment except that no neurotoxin was injected.

\section{Histology}

At the end of the experiment, the animals were killed using a lethal barbiturate overdose and perfused transcardially with $0.9 \%$ saline followed by $10 \%$ formaldehyde solution. The brains were stored in $10 \%$ formalin solution for at least $48 \mathrm{hr}$ and then transferred to a $25 \%$ sucrose-formalin solution before $40 \mu \mathrm{m}$ coronal sections were cut throughout the region of the nucleus accumbens. Alternate slices were stained using thionin. Slides were examined for placement and extent of the lesion; the latter was assessed by microscopically examining sections for areas of marked cell loss as well as general shrinkage of a region relative to sham controls.

\section{Procedure}

Training. After recovery from surgery, subjects were placed on a food deprivation schedule such that they received $15 \mathrm{gm}$ of their maintenance diet daily to maintain them at $\sim 85 \%$ of their free-feeding weight. The animals were fed each day after the training sessions. Animals were provided tap water ad libitum while in the home cage. Each session started with the illumination of the house light and insertion of the levers where appropriate and ended with the retraction of the levers and turning off of the house light. All sessions were $30 \mathrm{~min}$ in duration unless otherwise stated.

Magazine training. Initially, all subjects received two sessions of magazine training in which the pellet and sucrose outcomes were delivered on independent random time (RT) $60 \mathrm{sec}$ schedules with the levers withdrawn.

Lever training. The animals were next trained on random ratio (RR) schedules of reinforcement. Each lever was trained separately, and for half of the animals in each group, the left lever earned pellets and the right lever earned the sucrose solution. The remaining animals received the opposite action-outcome pairings. The animals first received $1 \mathrm{~d}$ of continuous reinforcement and were then shifted to an RR-5 schedule (i.e., each action delivered an outcome with a probability of 0.2). After $3 \mathrm{~d}$ of training this was changed to an RR-10 schedule (or a probability of 0.1 ) for $3 \mathrm{~d}$ and then to an RR-20 schedule (or a probability of 0.05 ) for an additional $3 \mathrm{~d}$ of training. The animals received two training sessions each day, one with each action-outcome pair. The animals had a break of at least 1 hr between sessions, and the order was alternated each day.

Devaluation extinction tests. After the final day of RR-20 training, all of the rats were given access ad libitum to one of the two outcomes for $1 \mathrm{hr}$ in the home cage. Half of the animals in each action-outcome pair assignment received pellets (50 gm placed in a bowl in the home cage), and the remaining animals received sucrose $(50 \mathrm{ml}$ in a drinking bottle fixed to the front of the home cage). Immediately after the prefeeding, the animals were placed in the operant chambers. A $10 \mathrm{~min}$ choice extinction test was then conducted in which both levers were extended and the number of presses was counted on each lever. No outcomes were delivered during the test. After the first devaluation test, the animals received $1 \mathrm{~d}$ of retraining (RR-20; one session for each action-outcome pair) and were then given a second devaluation test on the following day. The second test was identical to the first except that those animals that had had pellets devalued previously now had sucrose devalued and those that had had sucrose devalued were now prefed pellets.

Reward test. After the second devaluation test conducted in extinction, the animals were retrained (RR-20; one 30 min session for each actionoutcome pair) and on the following day tested for their sensitivity to the devaluation manipulation when performance was rewarded. This test was conducted in the same manner as the extinction test except that the outcomes were delivered as a consequence of instrumental performance. In this $20 \mathrm{~min}$ session, the two outcomes were delivered according to independent ratio schedules (RR-20).

\section{Experiment 2: contingency degradation}

\section{Subjects and apparatus}

The subjects and general apparatus are as described for experiment 1.

\section{Procedure}

Contingency degradation training. After the devaluation tests, the rats received $2 \mathrm{~d}$ of retraining on RR-20 schedules before the selective degradation of one of the instrumental contingencies. At the end of training, each lever press earned a unique outcome (pellets or a $20 \%$ sucrose solution) with a fixed probability $[p$ (outcome/action $)=0.05]$. The rats continued to be trained on the two actions with the appropriate paired outcomes, but in addition to being earned by one of the actions, one of the outcomes was now also delivered noncontingently with the same probability $[p$ (outcome/no action $)=0.05]$ in each second without a response. For one lever, the noncontingent or free reinforcer was the same as that earned by a response on that lever. Thus, the experienced probability of the delivery of that particular outcome was the same whether or not the animals performed that action, a procedure that should act to degrade that specific action-outcome contingency. For the other lever, the free reinforcer was different from the earned reinforcer, and so this contingency was not degraded. For half of the animals the lever press-pellet contingency was degraded, whereas for the remainder the lever press-sucrose contingency was degraded. The rats were given two 20 min training sessions each day, one on each lever with a break of $\sim 2 \mathrm{hr}$ between sessions. The order of the sessions was alternated each day and training continued for $8 \mathrm{~d}$.

Contingency degradation extinction test. On the day after the final day of contingency training, rats in both groups received a choice extinction test. The test began with the insertion of both levers and the onset of the house light and ended 10 min later with the retraction of the levers and the offset of the house light. No outcomes were presented during this session.

\section{Experiment 3: Pavlovian-instrumental transfer} Subjects and apparatus

The subjects and apparatus are as described for experiment 1 . 

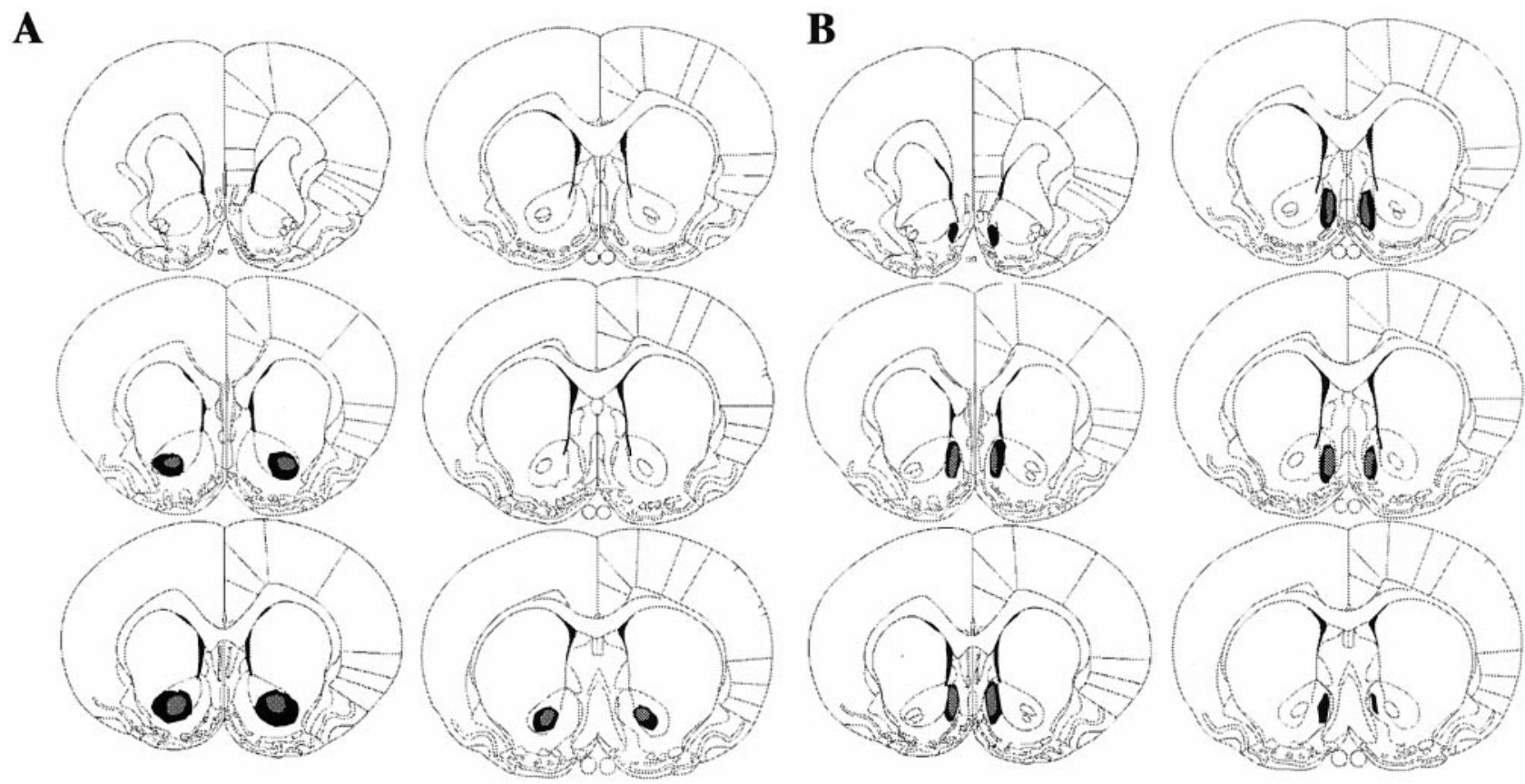

Figure 1. Schematic representation of excitotoxic lesions of the NAC core $(A)$ and shell $(B)$. Shaded areas represent the maximum (black) and minimum ( gray) extent of the lesions for the animals included in the behavioral analyses. Coronal sections are taken from the following points in the anteroposterior plane beginning at top left: $+2.2,+1.7,+1.6,+1.2,+1.0$, and $+0.7 \mathrm{~mm}$ anterior to bregma (Paxinos and Watson, 1998).

\section{Procedure}

Pavlovian training. Initially the animals were retrained for $3 \mathrm{~d}$ on RR-20 schedules after the contingency extinction test. Next the animals received eight sessions of Pavlovian conditioning. Two $80 \mathrm{~dB}$ auditory stimuli (tone and white noise) served as conditioned stimuli (CSs) and were paired with either pellet or sucrose delivery. For half of each lesion condition, the tone was paired with pellet delivery, and the noise was paired with sucrose delivery. The remaining half received the reverse pairings. Four presentations of each stimulus were given in each session in random order interspersed with periods in which no stimuli were present. The length of the intertrial intervals varied, but on average these intervals were $5 \mathrm{~min}$. The stimuli presentations were 2 min long during which the appropriate outcome was delivered on an RT $30 \mathrm{sec}$ schedule. The number of magazine entries during the stimuli as well as in a prestimulus interval of equal length $(2 \mathrm{~min})$ was measured. After $8 \mathrm{~d}$ of stimulus training, the animals were retrained with the levers for $1 \mathrm{~d}$ on the RR-20 schedule before testing.

Pavlovian-instrumental transfer test. The animals received two extinction tests (one on each lever) $1 \mathrm{~d}$ apart. During each test one of the levers was available, and each stimulus was presented four times interspersed with intervals of no stimulus $(\varnothing)$. Each test was $32 \mathrm{~min}$ in duration. In the first $8 \mathrm{~min}$ the levers were available, but no stimuli were presented. This period was followed by 12 bins of 2 min each and contained a total of eight stimulus trials [four tone trials $(\mathrm{T})$ and four noise trials $(\mathrm{N})$ intermixed with four $\varnothing$ trials in the following order: $\varnothing, \mathrm{T}, \mathrm{N}, \varnothing, \mathrm{N}, \mathrm{T}, \varnothing$, $\mathrm{N}, \mathrm{T}, \varnothing, \mathrm{T}$, and $\mathrm{N}]$.

\section{RESULTS}

\section{Experiment 1: outcome devaluation}

The aim of experiment 1 was to assess the impact of lesions of the NAC core or shell on instrumental learning by assessing the impact of these lesions on training and, subsequently, on the sensitivity of the performance of the rat to the effects of outcome devaluation using a specific satiety treatment (Adams and Dickinson, 1981; Balleine and Dickinson, 1998b). It has been shown that this treatment induces a selective reduction in the current incentive value of the prefed outcome (Balleine and Dickinson, 1998b), and so, in agreement with previous findings, we anticipated that sham rats would show an outcome devaluation effect reflected in fewer responses being performed on the lever that, in training, delivered the now-devalued outcome. However, if the NAC is required for either the encoding of the incentive value of an instrumental outcome or the formation of action-outcome associations during instrumental training, then any outcome devaluation effect established in sham controls should be severely attenuated in the lesioned groups.

\section{Histology}

No recovery problem or weight loss was observed after surgery. Figure 1 displays the maximum and minimum damage resulting from the lesions for the animals included in the behavioral analyses of the core $(A)$ and shell $(B)$ based on the stereotaxic atlas of the rat brain by Paxinos and Watson (1998). Any animals with unilateral damage or damage outside the target region were excluded from the behavioral analyses. Figure 2 displays photomicrographs taken of representative lesions of the sham (right images), core (top images), and shell (bottom images) groups. The photographs on the right in Figure 2 show magnifications of the lesions shown in the images on the left. Magnifications of the sham brain in the region of the core and shell are shown for comparison in the top right and bottom right images, respectively, of Figure 2. Core lesions (Fig. 2, top images) resulted in substantial neuronal loss in the core region bilaterally and typically extended in the anteroposterior direction from 0.7 to $1.7 \mathrm{~mm}$ anterior to bregma. Generally the lesion did not extend ventrally to the ventral pallidum or dorsally to the caudate-putamen. Any animals with marked damage to the NAC shell were excluded from the behavioral analysis. NAC shell lesions (Fig. 2, bottom images) destroyed neurons in the mediodorsal shell bilaterally and typically extended in the anteroposterior direction from 1.0 to $1.7 \mathrm{~mm}$ anterior to bregma. The ventral and ventrolateral portions of the NAC shell appeared unaffected by the lesions. Any animals with substantial damage of the NAC core or any other surrounding structures outside the shell were excluded. 

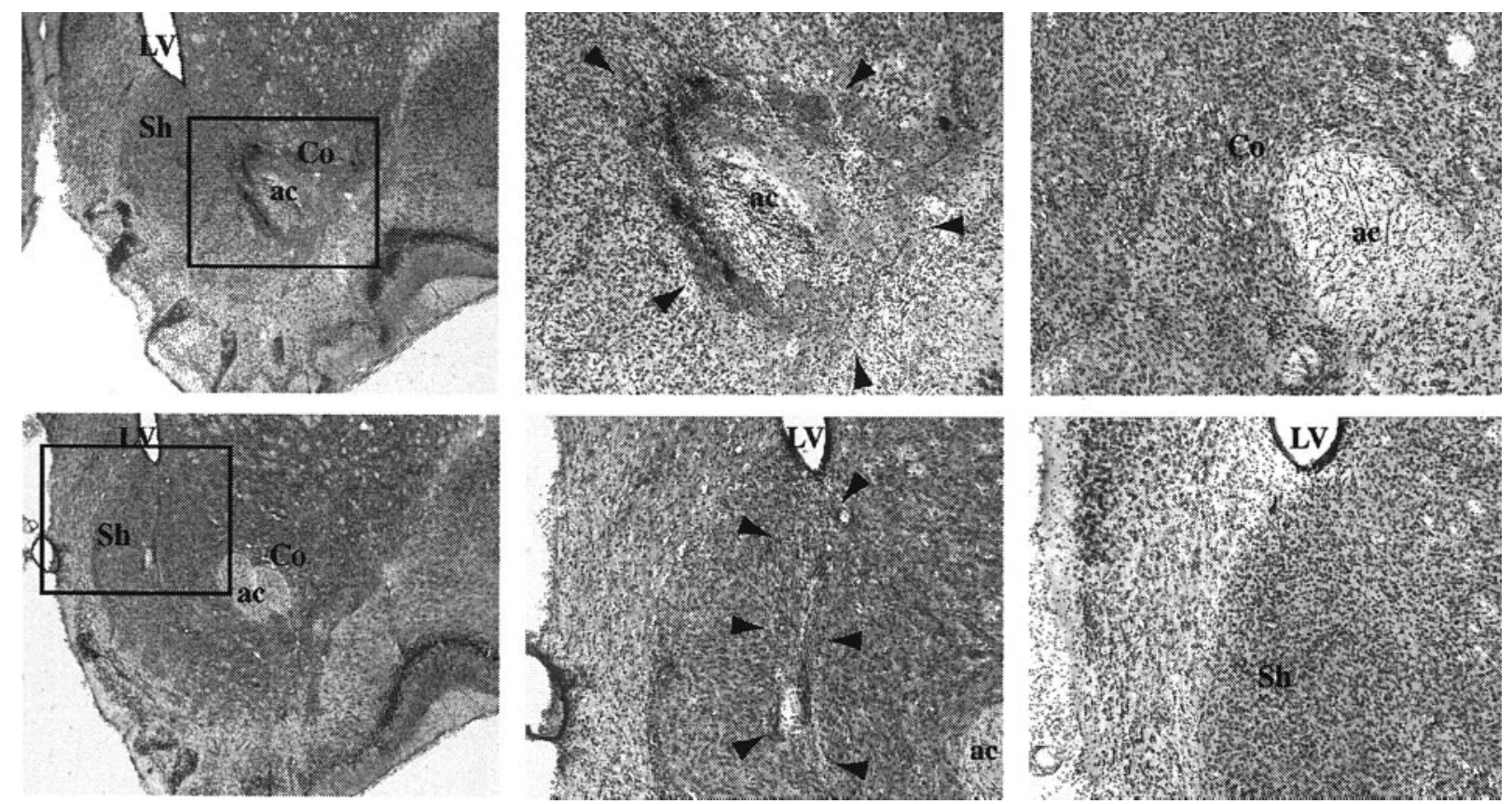

Figure 2. Photomicrographs showing thionin-stained coronal sections through the nucleus accumbens. Top, Representative core lesions. Bottom, Representative shell lesions. Left, Images of the general region of the NAC (40× magnification) of core-lesioned (top) and shell-lesioned (bottom) animals. Middle, Greater magnification of the region indicated by the outlined boxes in the left images; arrowheads indicate lesion boundaries. Right, High-magnification photographs of the region of the lesions shown in the middle images (in lesioned animals) but in sham-lesioned animals. All images are from slices taken at $\sim 2.6 \mathrm{~mm}$ anterior to bregma. ac, Anterior commissure; $C o$, NAC core; $L V$, lateral ventricle; $S h$, NAC shell.

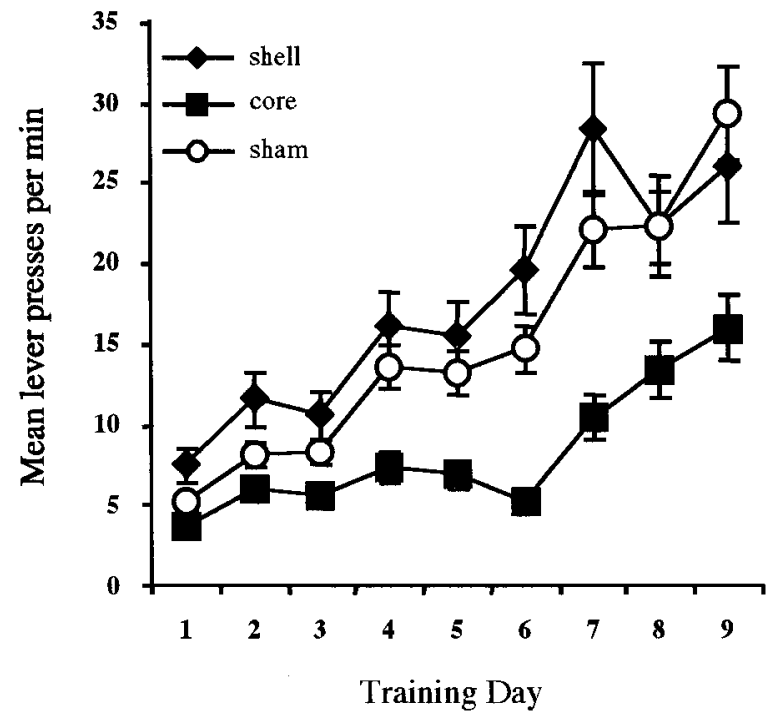

Figure 3. Mean lever-press responses per minute for the three groups across days of training. Responding was reinforced on days 1-3 on an RR-5 schedule of reinforcement, on days $4-6$ on an RR-10 schedule of reinforcement, and on days 7-9 on an RR-20 schedule of reinforcement.

\section{Training}

As shown in Figure 3, animals from each group acquired the lever-press response for the two outcomes, and their response rates increased as the ratio schedule parameter increased across days. Nevertheless, the groups clearly diverged in their performance during training; the core-lesioned rats performed at a generally lower rate and the shell-lesioned rats performed at a slightly higher rate than did the sham-lesioned rats. Initial statistical analysis revealed that there was no effect of outcome type
$(F<1)$, and so the data were collapsed across outcome for presentation and subsequent analysis. ANOVA revealed a significant effect of training day $\left[F_{(8,16)}=13.205 ; p<0.01\right]$. Also, as indicated by Figure 3, a significant effect of group confirms that the groups responded at different rates $\left[F_{(2,21)}=6.907 ; p<0.01\right]$. Additionally there was a marginal day $\times$ group interaction suggesting that, at least numerically, the increase in responding across days differed between the three groups $\left[F_{(16,168)}=1.655\right.$; $p=0.06]$. Tukey pairwise comparisons conducted on the mean rate of performance during training revealed that the corelesioned group responded at a reliably lower rate than did both the sham- and the shell-lesioned groups $(p<0.01)$. The numerical difference observed between the shell- and sham-lesioned groups failed to achieve significance $(p>0.05)$.

\section{Devaluation extinction tests}

Mean lever presses per minute during the choice extinction test are presented in Figure 4 collapsed across the two tests. Inspection of Figure 4 reveals a clear devaluation effect in both the sham- and shell-lesioned rats. However, the core animals show a very different pattern of responding, decreasing responding on both levers. A two-way ANOVA conducted using factors of group, separating the three lesion conditions, and of devaluation, separating performance on the lever that, in training, delivered the now-devalued outcome from responding on the other lever, revealed a significant main effect of group $\left[F_{(2,21)}=6.223\right.$; $p<0.01]$, an effect of devaluation $\left[F_{(1,21)}=26.696 ; p<0.01\right]$, and more important, a significant group $\times$ devaluation interaction $\left[F_{(1,21)}=4.821 ; p<0.05\right]$. Simple effects analyses revealed that, although a significant effect of devaluation emerged in both the sham-lesioned $\left[F_{(1,21)}=24.581 ; p<0.01\right]$ and shell-lesioned $\left[F_{(1,21)}=13.244 ; p<0.01\right]$ rats, core-lesioned rats did not respond differently on the two levers; thus whereas the devaluation 


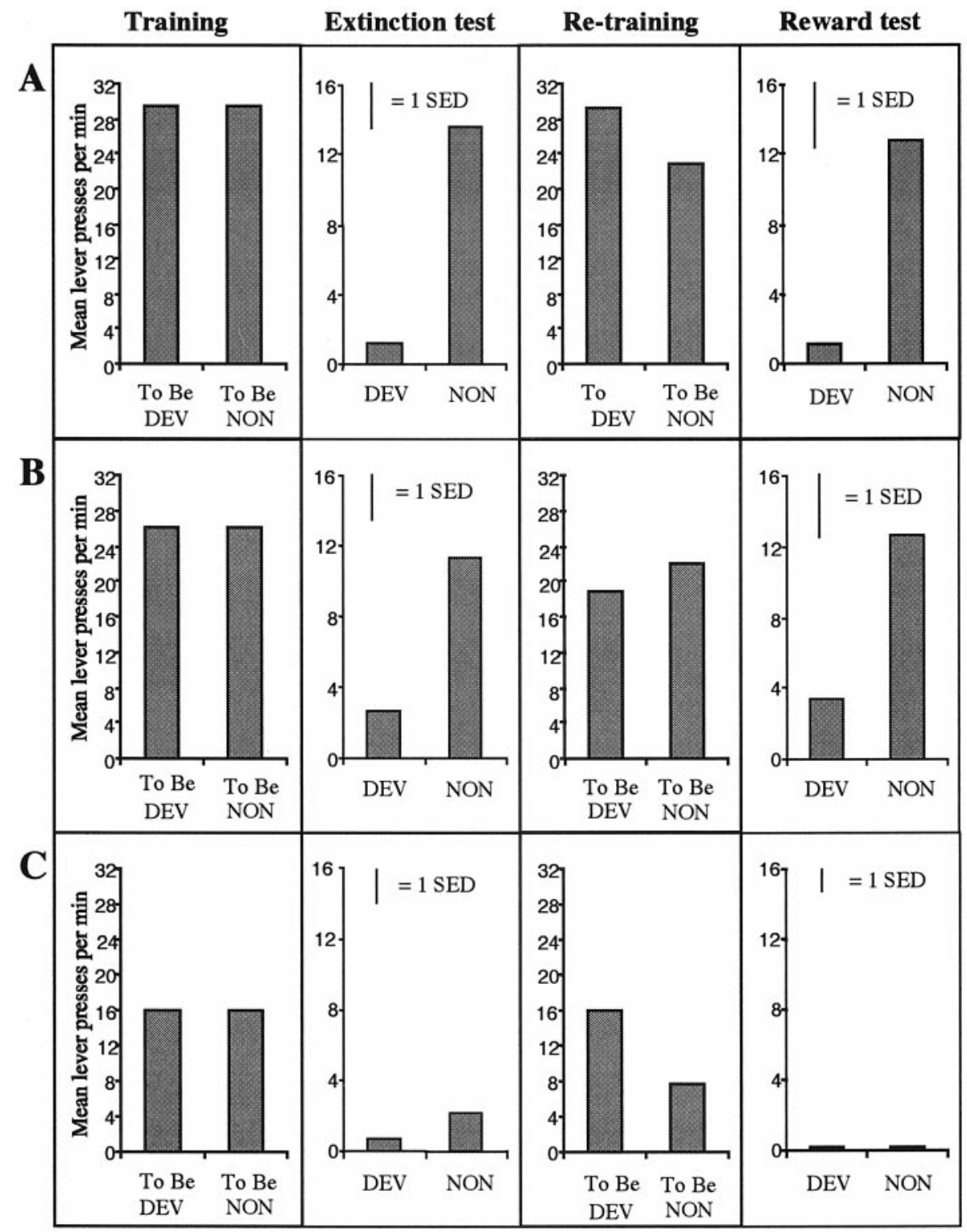

Figure 4. Mean lever-press responses for the devaluation tests conducted in experiment 1 for each of the lesioned groups. $A$, Sham. $B$, Shell. $C$, Core. For $A-C$, the left panel displays the mean response rate per minute at the end of training. The second panel from the left displays responses per minute for the devalued and nondevalued outcomes in a two-lever, choice extinction test. The third panel from the left illustrates the mean performance during the retraining sessions, and the right panel displays the mean lever-press responses per minute in a rewarded, two-lever test after devaluation of one of the instrumental outcomes. SED represents the SE of the difference in responding for the within-subjects variable. $D E V$, Devalued; $N O N$, nondevalued. manipulation clearly affects their lever-press performance, the core-lesioned animals fail to show a selective devaluation effect $\left[F_{(1,21)}<1\right]$.

\section{Reward test}

The failure of core-lesioned rats to show a selective devaluation effect could be explained in several ways. It could be that the core region of the accumbens is critical for encoding the value of different outcomes, or alternatively, it could be that the memory of the two outcomes is impaired. If animals are unable to differentiate the two outcomes in extinction, then devaluation of one outcome may generalize to the other resulting in a decrease in performance of both responses. To assess whether the deficit observed in the extinction test was caused by a failure to discriminate the outcomes in extinction rather than in devaluation per se, the animals were retrained and tested again for their sensitivity to outcome devaluation but this time with the two outcomes being delivered. Because the two outcomes are delivered in this test, any deficit observed in the core group cannot be attributed to failure to remember and distinguish the two outcomes but, rather, would suggest a deficit in the instrumental incentive processes that mediate outcome devaluation. The results of this test are presented in the right panels of Figure 4. Generally, the pattern of results in the reward test was similar to those results observed in the extinction test; sham- and shell-lesioned rats showed strong selective devaluation effects, whereas core-lesioned rats failed to show any selective effect of the devaluation treatment. The statistical analysis revealed significant main effects of devaluation $\left[F_{(1,21)}=9.752 ; p<0.01\right]$ and group $\left[F_{(2,21)}=5.205 ; p<0.05\right]$, and again, simple effects analyses found that, although the devaluation effect was reliable in the sham-lesioned $\left[F_{(1,21)}=9.447\right.$; $p<0.01]$ and shell-lesioned $\left[F_{(1,21)}=6.171 ; p<0.01\right]$ rats, even when the outcomes were delivered, the core-lesioned rats failed to show a selective devaluation effect $\left[F_{(1,21)}<1\right]$ and performed very few responses on either lever. This suggests that the deficit in the core animals, observed both when tested in extinction and when performance is rewarded, is caused by a failure of the current incentive values of the instrumental outcomes to control performance selectively. Clearly the incentive value of the non- 
devalued outcome was unable to maintain instrumental responding in the core-lesioned group.

In addition to leaning the value of the instrumental outcome, considerable evidence suggests that, in instrumental conditioning, rats can encode the specific action-outcome relations to which they are exposed during training. Furthermore, numerous findings suggest that, in intact animals, instrumental performance is sensitive to the contingency or causal relationship between performance of an action and delivery of its specific outcome (Balleine and Dickinson, 1998a; Corbit and Balleine, 2000).

With this in mind, one possible explanation of the low responding observed in core-lesioned animals both in training and particularly after outcome devaluation in experiment 1 is that these lesions render rats unable to encode specific action-outcome associations, and as such, changes in the value of one outcome produced a general rather than a selective reduction in lever-press performance on the test. This possibility is examined in experiment 2.

\section{Experiment 2: contingency degradation}

The rats received sessions in which the contingency between one action and its outcome on which they were trained was selectively degraded while the other action-outcome contingency remained intact [details of the procedure used have been reported elsewhere (Corbit and Balleine, 2000)]. After this training, the rats were given a test in extinction on the two levers to assess the impact of the shift in contingency. In agreement with previous findings, it was predicted that sham animals should perform fewer responses on the lever for which the action-outcome contingency has been degraded relative to the other lever. If, however, the deficits observed in experiment 1 reflect an impairment in the ability of core-lesioned rats to encode specific action-outcome contingencies, then core-lesioned rats should fail to show this effect; i.e., they should not show a selective decrease in performance after degradation of one action-outcome contingency and should perform both responses at comparable rates in the extinction test.

\section{Contingency degradation training}

The left panels of Figure 5 depict the effects of contingency degradation training across days. ANOVA revealed a significant main effect of group $\left[F_{(2,21)}=6.107 ; p<0.01\right]$, suggesting that, as in experiment 1 , rates of responding differed between the three groups and suggesting an effect of day $\left[F_{(7,147)}=2.996 ; p<0.01\right]$ and, importantly, an effect of contingency $\left[F_{(1,21)}=9.117 ; p<\right.$ $0.01]$ indicating that, overall, rats reduced responding on the lever for which the contingency had been degraded. Furthermore, although a reliable day $\times$ contingency interaction emerged $\left[F_{(7,147)}=3.434 ; p<0.01\right]$, indicating that the contingency effect increased across days of training, no other interactions were significant $(F<1)$, confirming that a selective effect of contingency degradation emerged in each of the lesion groups. Importantly, although performance in the core-lesioned rats was lower than that in the sham- and shell-lesioned groups, they showed an effect of contingency degradation across days, an effect that persisted in the final extinction test (Fig. 5, right panels). In general, therefore, it appears that all three groups were sensitive to degradation of the action-outcome contingency and selectively reduced performance accordingly.

\section{Contingency extinction test}

As indicated by the right panels of Figure 5, the effect of contingency degradation found in training persisted in the test con-
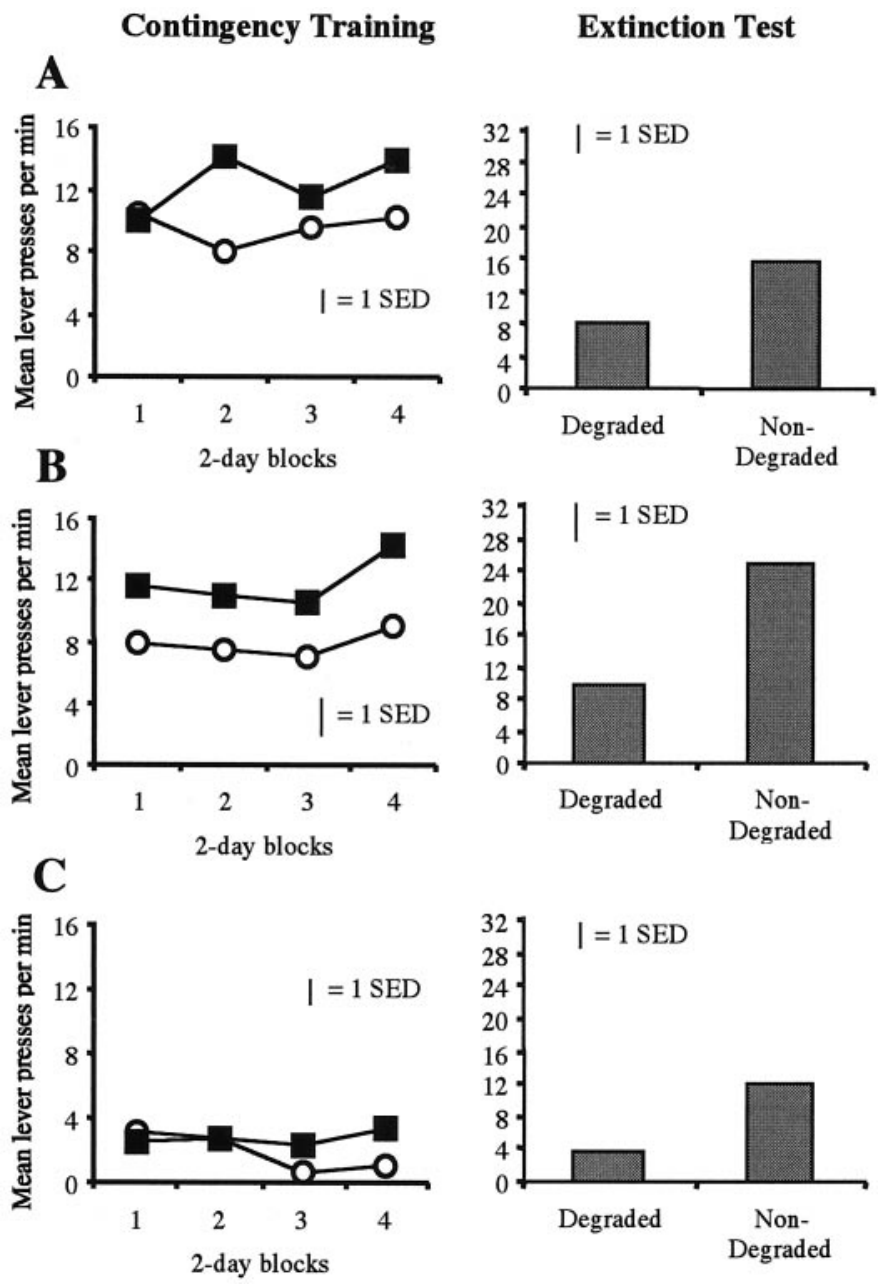

Figure 5. Mean lever-press responses during tests of the sensitivity of the animals to the selective degradation of one instrumental action-outcome contingency in each of the lesioned groups. $A$, Sham. $B$, Shell. $C$, Core. For $A-C$, the left panel displays mean lever presses per minute across days of contingency degradation training, and the right panel displays the mean responses per minute on the two levers in an extinction test.

ducted in extinction; i.e., the rats in each group performed fewer responses on the lever for which the instrumental contingency had been degraded. ANOVA found a significant effect of group $\left[F_{(2,27)}=3.664 ; p<0.05\right]$ and of contingency $\left[F_{(1,21)}=20.661 ;\right.$ $p<0.01]$, but, again, the group $\times$ contingency interaction was not reliable $\left[F_{(2,21)}=1.123 ; p>0.05\right]$. This finding adds additional weight to the argument that, although the overall rate of responding differed between the lesioned groups, degradation of one instrumental contingency was effective in each of the groups. As such, it is clear that neither core nor shell lesions affected the ability of the rats to encode the specific action-outcome associations to which they were exposed during training. These data suggest that the effects of core lesions on the devaluation tests in experiment 1 are unlikely to have been secondary to a deficit in instrumental learning produced by the lesion.

\section{Experiment 3: Pavlovian-instrumental transfer}

The results of experiment 1 suggest that the instrumental performance of animals with lesions of the NAC core is not sensitive to selective devaluation of one outcome although the results of experiment 2 provide evidence that these same rats can encode 

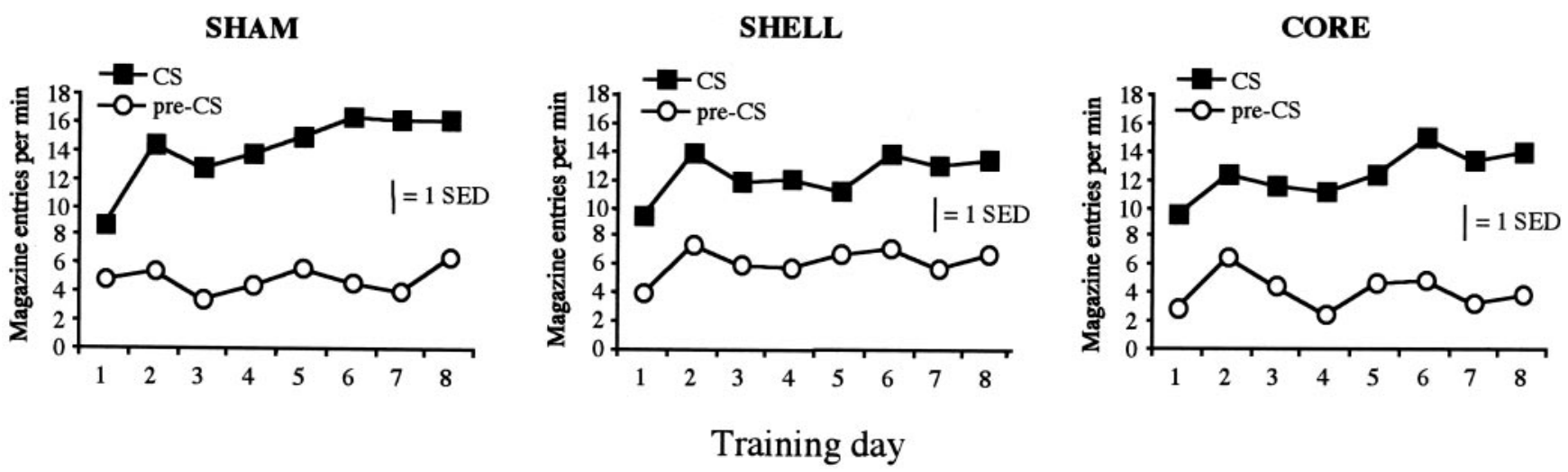

Figure 6. Mean magazine entries per minute during CS presentations and in the pre-CS interval across days of Pavlovian training.

the specific action-outcome contingencies to which they were exposed during training. Taken together, these results suggest that the NAC core, although not involved in the process by which rats encode action-outcome associations, is critically involved in the process via which the reward or incentive value of the instrumental outcome acts to control instrumental performance.

Contrary to this view, it has occasionally been suggested that outcome devaluation effects reflect a reduction in the excitatory effects of Pavlovian stimulus-outcome associations on instrumental performance (Rescorla and Solomon, 1967; Bindra, 1974, 1978). In general, these two-process accounts suppose that, in the course of instrumental training, stimuli that are present in the training situation become associated with the delivery of the instrumental outcome, and as a result of an excitatory relationship with reward, these stimuli can act to increase appetitive arousal, providing a source of motivation capable of modulating instrumental performance. To the extent that different cues predict different outcomes (e.g., the sight of the lever or the sound of the pellet dispenser or the sucrose pump), these stimulus-outcome associations could act to modulate responding selectively and so, at least potentially, to produce the differential performance observed in the outcome devaluation tests described in experiment 1 . From this perspective, rather than affecting the impact of the incentive value of the instrumental outcome on performance, lesions of the NAC core may affect instrumental performance by reducing the excitatory impact of Pavlovian cues.

In support of this suggestion, Pavlovian cues have been reported to influence the performance of independently trained instrumental actions, and numerous studies have reported that, in hungry animals, the presentation of a stimulus paired previously with food in a Pavlovian-conditioning phase can increase ongoing instrumental responding (Colwill and Rescorla, 1988; Balleine, 1994), an effect referred to as Pavlovian-instrumental transfer. In addition to having a generally arousing impact on performance, it has also been shown that specific cues can selectively enhance one but not another action because of a shared outcome (Colwill and Motzkin, 1994). Results such as these have been interpreted as suggesting that Pavlovian and instrumental conditioning share a common reinforcement mechanism and, hence, that Pavlovian and instrumental incentive processes are one and the same (cf. for review, see Bindra, 1974, 1978).

Critical tests of this suggestion have proven difficult to devise, but one prediction of relevance to the current study is that, on the basis of the effect of core lesions on instrumental outcome devaluation in experiment 1 , lesions of the NAC core should also produce a deficit in Pavlovian-instrumental transfer. As such, the purpose of experiment 3 was to examine whether the lesions that eliminated the specificity of outcome devaluation in experiment 1 would also affect the ability of Pavlovian cues to modulate instrumental performance in a Pavlovian-instrumental transfer test similar to that used by Colwill and Motzkin (1994).

In agreement with previous findings, we anticipated that the sham-lesioned rats would show positive transfer such that in the presence of a stimulus they would respond more on the lever that, in training, delivered the same outcome that had been paired previously with that stimulus during Pavlovian conditioning. If a common process mediates transfer and outcome devaluation, then shell-lesioned rats should be predicted to respond similarly to shams, whereas core-lesioned rats should show little or no selective transfer on the test.

\section{Pavlovian training}

To assess whether animals learned about the relationship between the stimuli and food presentations, the number of magazine entries during the stimuli was compared with the number of entries in the prestimulus interval. The training data are displayed in Figure 6, inspection of which suggests that Pavlovian training was similar in the three groups. Preliminary analysis suggested that there was no effect of stimulus type (noise vs tone; $F<1$ ), and so the data are presented collapsed across stimulus. ANOVA revealed a significant effect of interval (stimulus vs prestimulus) $\left[F_{(1,20)}=127.3 ; p<0.01\right]$, confirming that the rats made more magazine entries during the stimuli than in the prestimulus intervals, and a significant effect of training day $\left[F_{(7,140)}=8.765 ; p<0.01\right]$, suggesting that as training proceeded the animals entered the magazine more during the stimuli. There was also a significant interval $\times$ day interaction $\left[F_{(7,140)}=4.690\right.$; $p<0.01]$, indicating that the difference between stimulus and prestimulus intervals increased over days. There was, however, no effect of group, and none of the interactions involving groups approached significance (all $F$ values $<1$ ). There is no evidence in this experiment, therefore, that the acquisition of Pavlovian conditioning was affected by lesions of either the NAC core or shell.

\section{Pavlovian-instrumental transfer tests}

The objective of this experiment was to assess the impact of Pavlovian cues for reward on instrumental performance, and so for the purpose of analysis, the number of lever presses during the baseline or no-stimulus $(\varnothing)$ period was subtracted from the num- 


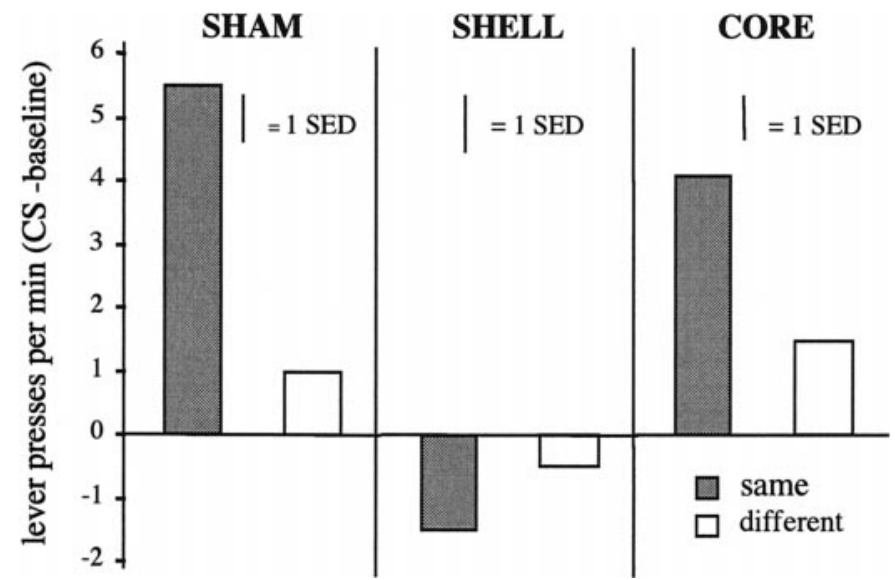

Figure 7. Mean lever presses per minute during the presentation of each of the stimuli minus the mean lever presses per minute during the no-stimulus $(\varnothing)$ baseline period for each of the three lesion groups. The term same refers to responding during the stimulus that was paired with the same outcome as that earned in training on that lever, whereas the term different refers to responding during the stimulus that was paired with a different outcome than that earned in training on that lever.

ber of lever presses performed during each of the stimuli. Positive transfer is indicated, using this measure, by positive numbers, whereas reduced transfer should be reflected as numbers close to zero. The data from the transfer tests are displayed in Figure 7.

It is clear from Figure 7 that a strong and selective positive transfer effect emerged in both the sham- and core-lesioned groups with responding on the lever that, in training, delivered the same outcome signaled by the stimulus being elevated more than responding on the other lever. In contrast, no positive transfer emerged in the shell-lesioned group. The statistical analysis confirmed this description; analysis of the test data revealed no effect of group $\left[F_{(2,20)}=2.568 ; p>0.05\right]$ but did find a significant effect of stimulus (i.e., same vs different) $\left[F_{(1,2)}=\right.$ 13.379; $p<0.01$ ] and, critically, a significant stimulus $\times$ group interaction $\left[F_{(2,20)}=6.257 ; p<0.01\right]$. Simple effects analyses revealed that, whereas there was a significant effect of stimulus in both the sham-lesioned $\left[F_{(1,20)}=22.154 ; p<0.01\right]$ and corelesioned $\left[F_{(1,20)}=3.288 ; p<0.05\right]$ groups, there was no stimulus effect in the shell-lesioned animals $\left[F_{(1,20)}<1\right]$.

These data strongly oppose the suggestion that outcome devaluation effects can be explained in terms of the excitatory impact of Pavlovian signals for reward on performance and suggest that the effects of core lesions induced in experiment 1 were not produced by any deficit in either Pavlovian conditioning or the excitatory impact of Pavlovian cues on instrumental performance. This conclusion is further supported by the striking deficit in transfer induced by shell lesions, a lesion that was found in experiment 1 to have no impact whatever on the sensitivity of the instrumental performance of the rat to the effects of outcome devaluation.

\section{DISCUSSION}

The results of this series of studies have important implications both for theories of accumbens function and, more generally, for theories of instrumental conditioning. With respect to the latter, it has long been argued that both primary incentives, such as foods and fluids, and Pavlovian-conditioned stimuli that predict those incentives affect instrumental performance via a common reward process (Rescorla and Solomon, 1967; Bindra, 1974,
1978). In contrast to this position, contemporary theories have drawn a strong distinction between these two sources of reward arguing, instead, that the incentive value of the instrumental outcome and the excitatory effects of Pavlovian cues on instrumental performance are mediated by distinct learning processes (Dickinson and Balleine, 1994; Balleine, 2000). The current results provide strong evidence of this latter position. Thus, although sham- and shell-lesioned animals show a clear selective devaluation effect, lesions localized to the accumbens core were found to eliminate the ability of the incentive value of different outcomes to control performance selectively after a specific satiety devaluation procedure. Additionally, core lesions did not appear to affect the excitatory influence of Pavlovian cues on instrumental performance. Together, consideration of the pattern of behavior on all tests must point to a specific deficit in the ability of the current value of different outcomes to control performance selectively after lesions of the NAC core.

In contrast, lesions of the accumbens shell had no detectable effect on outcome devaluation but had a clear and striking effect on the excitatory influence of Pavlovian cues on instrumental performance, eliminating the Pavlovian-instrumental transfer effect observed in sham- and core-lesioned rats. As such, this study provides evidence that instrumental and Pavlovian incentive learning processes are independent and, furthermore, that these incentive processes differentially involve accumbens core and shell, respectively.

A possible concern may be the somewhat depressed levels of responding in core-lesioned animals observed in training. However, a comparison of the performance of these animals on the last day of lever training and their performance in the devaluation tests suggests that their behavior on the test cannot be explained by a simple performance deficit. These animals markedly decrease responding after the devaluation treatment and additionally return to reasonable response levels in retraining sessions, suggesting that it is the experimental manipulation rather than a baseline effect that accounts for their low levels of responding. Importantly, the same animals show normal sensitivity to the degradation of one instrumental contingency despite their overall lower levels of responding. This finding replicates the results of Balleine and Killcross (1994) who also observed that lesions of the NAC depressed overall response rates while not having any effect on sensitivity to changes in the instrumental contingency. Balleine and Killcross (1994) did not, however, observe any effects of their lesions on performance after shifts in the motivational state of the animals, suggesting intact incentive learning. However, the behavioral procedures as well as the lesions were different in that study and may not have been sensitive or selective enough to detect effects like those observed in the current study. Further study will be required to evaluate this apparent discrepancy properly.

The argument that accumbens core and shell mediate distinct functions in instrumental conditioning is consistent with other recent theories of accumbens function advanced on the basis of studies assessing the intrinsic neuroanatomy and connectivity of the accumbens (Pennartz et al., 1994; Wright and Groenewegen, 1995). Indeed, in recent reviews Zahm (1999, 2000) has argued persuasively that core and shell regions represent rather different forms of neural processing proposing the existence of discrete neural networks involving these structures. Although these networks are complex and their function is only beginning to be understood, at least as applied to the neural structures mediating instrumental conditioning, a degree of consensus appears to be 
emerging. Thus, in agreement with the effects of core lesions in the current study, the administration of AP-5 into the accumbens core has been found to retard acquisition of the instrumental response (Kelley et al., 1997). Additionally, administration of 6-OHDA into the core has been shown to reduce instrumental performance (Sokolowski and Salamone, 1998). Kelley and colleagues have argued that this effect on the acquisition of the instrumental response after administration of AP-5 implicates the accumbens core in instrumental learning. Nevertheless, the finding in experiment 2 that, despite reduced response levels, sensitivity to selective degradation of the instrumental contingency remains primarily intact in core-lesioned rats suggests otherwise. It appears that the acquisition of specific action-outcome associations is mediated by structures afferent to the accumbens but that, nevertheless, the core region plays a key role in selecting or initiating actions based on the reward value of their consequences. This view is similar to one recently advanced by Sokolowski and Salamone (1998) and helps to clarify the source of the lack of selectivity in outcome devaluation observed in experiment 1 . Recall that, although only one of the two instrumental outcomes was devalued in each of the extinction tests conducted in experiment 1 , rats with lesions of the core, although manifestly sensitive to devaluation per se, failed to distinguish in their performance between the action that delivered the devalued outcome in training from the action that delivered previously the nondevalued outcome. Such a result should be anticipated, however, if it is via the core region that the current incentive value of the instrumental outcome acts to modulate performance.

In the current study lesions of the shell produced very different effects on instrumental conditioning, having no effect on either outcome devaluation or the selective degradation of the instrumental contingency in experiments 1 and 2 but eliminating Pavlovian-instrumental transfer in experiment 3 . Several previous reports have suggested that the shell region is responsive to signals for reward (Johnson et al., 1995; Bassareo and DiChiara, 1997), and indeed, lesions of the shell have been found to attenuate lever pressing for stimuli paired previously with reward (Parkinson et al., 1999). As such, these findings suggest that the shell region may mediate the impact of stimulus-reward associations on instrumental performance, a suggestion for which we found direct evidence in experiment 3. After the pairings of two stimuli with either the pellet or sucrose outcome used in instrumental training, the effect of these stimuli on lever-press performance was assessed in a transfer test. Both the sham- and the core-lesioned rats showed strong positive transfer; i.e., the stimuli selectively elevated responding on the lever that, in training, had delivered the same outcome that was signaled by that stimulus. Importantly, this effect was not evident in the shell-lesioned rats, suggesting that the lesion primarily abolished the excitatory influence of Pavlovian stimuli on performance. This effect does not appear to be because of a deficit in Pavlovian conditioning in the shell-lesioned rats, nor, in view of their performance in experiments 1 and 2, was it a consequence of their inability to recall what outcome had been trained with the particular action. Rather, it appears that it was the interaction of Pavlovian and instrumental learning that was affected by the lesion.

The finding that lesions of the accumbens shell induce a deficit in Pavlovian-instrumental transfer without affecting either Pavlovian or instrumental conditioning per se suggests that the shell may be a central structure through which feedback from cues associated with reward helps to activate and guide actions that are instrumental to gaining access to basic commodities. Again the work of Zahm $(1999,2000)$ is of interest in this context. This author notes that the rich projections from the shell to the ventromedial pallidum appear to provide the basis for feedback from the accumbens shell to cortical structures thought to be involved in executive and premotor functions, such as the prefrontal cortex, and to have strong projections back to the core region of the accumbens. Because current evidence suggests that the prelimbic region of the prefrontal cortex is strongly involved in the formation of the instrumental action-outcome association (Balleine and Dickinson, 1998a), this feedback loop would appear to provide a ready means by which Pavlovian cues could affect instrumental performance via the output of the core to other basal ganglia structures.

In summary, the current results provide evidence of a functional dissociation in the involvement of the NAC core and shell in instrumental conditioning and offer additional support for the existence of two independent, interacting neural networks involving these subregions. One network involving the core appears to be involved in mediating the impact of evaluative processes via which animals encode the incentive value of the instrumental outcome on the performance of goal-directed actions. The second network, involving the accumbens shell, appears to be involved in mediating the excitatory effects of stimuli that anticipate reward on goal-directed performance.

\section{REFERENCES}

Adams CD, Dickinson A (1981) Instrumental responding following reinforcer devaluation. Q J Exp Psychol 33B:109-122.

Alheid GF, Heimer L (1988) New perspectives in basal forebrain organization of special relevance for neuropsychiatric disorders: the striatopallidal, amygdaloid, and corticopetal components of substantia innominata. Neuroscience 27:1-39.

Balleine BW (1994) Asymmetrical interactions between thirst and hunger in Pavlovian-instrumental transfer. Q J Exp Psychol B 47:211-231.

Balleine BW (2000) Incentive processes in instrumental conditioning. In: Handbook of contemporary learning theories (Mowrer R, Klein S, eds), pp 307-365. Hillsdale, NJ: LEA.

Balleine BW, Dickinson A (1998a) Goal-directed instrumental action: contingency and incentive learning and their cortical substrates. Neuropharmacology 37:407-419.

Balleine BW, Dickinson A (1998b) The role of incentive learning in instrumental outcome revaluation by sensory-specific satiety. Anim Learn Behav 26:46-59.

Balleine BW, Killcross S (1994) Effects of ibotenic acid lesions of the nucleus accumbens on instrumental action. Behav Brain Res 65:181-193.

Bassareo V, DiChiara G (1997) Differential influence of associative and non-associative learning mechanisms on the responsiveness of prefrontal and accumbal dopamine transmission to food stimuli in rats fed $a d$ libitum. J Neurosci 17:851-861.

Bindra D (1974) A motivational view of learning, performance, and behavior modification. Psychol Rev 81:199-213.

Bindra D (1978) How adaptive behavior is produced: a perceptualmotivational alternative to response-reinforcement. Behav Brain Sci 1:41-91.

Colwill RM, Motzkin DK (1994) Encoding of the unconditioned stimulus in Pavlovian conditioning. Anim Learn Behav 22:384-394.

Colwill RM, Rescorla RA (1986) Associative structures in instrumental conditioning. In: The psychology of learning and memory, Vol 20 (Bower GH, ed), pp 55-104. New York: Academic.

Colwill RM, Rescorla RA (1988). Associations between the discriminative stimulus and the reinforcer in instrumental learning. J Exp Psychol Anim Behav Process 14:155-164.

Corbit LH, Balleine BW (2000) The role of the hippocampus in instrumental conditioning. J Neurosci 20:4233-4239.

Dickinson A, Balleine BW (1994) Motvational control of goal-directed action. Anim Learn Behav 22:1-18.

Groenewegen HJ, Russchen FT (1984) Organization of the efferent projections of the nucleus accumbens to pallidal, hypothalamic, and mesencephalic structures: a tracing and immunohistochemical study in the cat. J Comp Neurol 23:103-120.

Haber SN, Groenewegen HJ, Grove EA, Nauta WJH (1985) Efferent connections of the ventral pallidum: evidence of a dual striatopalliofugal pathway. J Comp Neurol 235:322-335.

Johnson PI, Goodman JB, Condon R, Stellar JR (1995) Reward shifts 
and motor responses following microinjections of opiate-specific agonists into either the core or shell of the nucleus accumbens. Psychopharmacology (Berl) 120:195-202.

Kelley A, Smith-Row SL, Holahan MR (1997) Response-reinforcement learning is dependent upon $N$-methyl-D-aspartate receptor activation in the nucleus accumbens core. Proc Natl Acad Sci USA 94:12174-12179.

Kelley AE, Domesick VB (1982) The distribution of the projections from the hippocampal formation to the nucleus accumbens in the rat: an anterograde and retrograde horseradish peroxidase study. Neuroscience 7:2321-2325.

Kelley AE, Domesick VB, Nauta WJH (1982) The amygdalostriatal projection in the rat: an anatomical study by anterograde and retrograde tracing methods. Neuroscience 7:615-630.

Mogenson GJ, Yim CY (1991) Neuromodulatory functions of the mesolimbic dopamine system: electrophysiological and behavioral studies. In: The mesolimbic dopamine system: from motivation to action (Willner P, Scheel-Kruger J, eds). New York: Wiley.

Mogenson GJ, Jones DL, Yim CY (1980) From motivation to action: functional interface between the limbic system and the motor system. Prog Neurobiol 14:69-97.

Mogenson GJ, Yang CR, Yim CY (1988) Influence of dopamine on limbic inputs to the nucleus accumbens. In: The mesocorticolimbic system (Kalivas PW, Nemeroff CB, eds), pp 86-100. New York: New York Academy of Sciences.

Moore RY, Bloom FE (1978) Central catecholamine neuron systems: anatomy and physiology of the dopamine systems. Annu Rev Neurosci 1:129-169.

Nauta WJ, Domesick VB (1984) Afferent and efferent relationships of the basal ganglia. Ciba Found Symp 107:3-29.

Parkinson JA, Olmstead MC, Burns LH, Robbins TW, Everitt BJ (1999) Dissociation in effects of lesions of the nucleus accumbens core and shell on appetitive Pavlovian approach behavior and the potentiation of conditioned reinforcement and locomotor activity by D-amphetamine. J Neurosci 19:2401-2411.

Paxinos G, Watson C (1998) The rat brain in stereotaxic coordinates. San Diego: Academic.

Pennartz CMA, Groenewegen HJ, Lopes de Silva FH (1994) The nucleus accumbens as a complex of functionally distinct neuronal ensembles: an integration of behavioural, electrophysiological, and anatomical data. Prog Neurobiol 42:719-761.

Powell EW, Leman RB (1976) Connections of the nucleus accumbens. Brain Res 105:389-403.

Rescorla RA, Solomon RL (1967) Two-process learning theory: relationship between Pavlovian conditioning and instrumental learning. Psychol Rev 74:151-182.

Robbins TW, Everitt BJ (1996) Neurobehavioral mechanisms of reward and motivation. Curr Opin Neurobiol 6:228-236.

Sokolowski JD, Salamone JD (1998) The role of accumbens dopamine in lever pressing and response allocation: effects of 6-OHDA injected into core and dorsomedial shell. Pharmacol Biochem Behav 59:557-566.

Wright CI, Groenewegen HJ (1995) Patterns of convergence and segregation in the medial nucleus accumbens of the rats: relationships of prefrontal cortical, midline thalamic and basal amygdaloid afferents. J Comp Neurol 361:383-403.

Zahm DS (1999) Functional-anatomical implications of the nucleus accumbens core and shell subterritories. Ann NY Acad Sci 877:113-128.

Zahm DS (2000) An integrative neuroanatomical perspective on some subcortical substrates of adaptive responding with emphasis on the nucleus accumbens. Neurosci Biobehav Rev 24:85-105.

Zahm DS, Brog JS (1992) On the significance of subterritories in the "accumbens" part of the rat ventral striatum. Neuroscience 50:751-767. 